\title{
Erratum
}

\section{Semi-Infinite Weil Complex and the Virasoro Algebra}

\author{
Boris Feigin $^{1}$ and Edward Frenkel ${ }^{2}$ \\ 1 Landau Institute for Theoretical Physics, Moscow, Russia \\ 2 Department of Mathematics, Harvard University, Cambridge, MA 02138, USA
}

Received February 21, 1992

Commun. Math. Phys. 137, 617-639 (1991)

In Sect. 4, on p. 636, we had assumed an incorrect structure of the Fock representations $\mathscr{H}_{p}$ of the Virasoro algebra, taken from [1], Theorem 1.10 (cf. Fig. 5). In fact, the module $\mathscr{H}_{p}$ is isomorphic to the Verma module, if $p \leqq 0$, and to the contragradient Verma module, if $p>0$, with highest weight $h_{p}^{\prime}=-(p-2)(p+1) / 2$ and central charge $28[2]$.

For this reason the exact sequences (24), (25), and Proposition 7 on p. 637 are also incorrect. Proposition 7 should read as follows.

Proposition 7. 1) Let $m \geqq 0, n \leqq 0$ be of equal parity. Then $h^{j}\left(L_{m} \otimes \mathscr{H}_{n}\right)=\delta_{j, 0}$, if $m=-n$ and 0 , otherwise.

2) Let $m \geqq 0, n \leqq 0$ be of different parity. Then $h^{j}\left(L_{m} \otimes \mathscr{H}_{n}\right)=\delta_{j, 1}$, if $m=-n-1$ and 0 , otherwise.

3) Let $m \geqq 0$ and $0<n<m$. Then $h^{j}\left(L_{m} \otimes \mathscr{H}_{n}\right)=0$ for any $j$.

Proof of parts 1) and 2) follows from the isomorphism $\mathscr{H}_{n} \simeq M_{\left(k_{n}^{\prime}, 28\right)}$ for $n \leqq 0$, Proposition 5, and the short exact sequence

$$
0 \rightarrow L_{\chi} \rightarrow M_{\chi}^{*} \rightarrow M_{\chi 1}^{*} \rightarrow 0 .
$$

Part 3) can be proved in a similar fashion.

This corrects (and simplifies) the statement of Theorem 1 on p. 628.

Theorem 1. 1) Let $p=-2 m, m \geqq 0$. Then $h_{p}^{0, l}=1$, if $l \leqq m$, and $h_{p}^{j, l}=0$, otherwise.

2) Let $p=-2 m-1, m \geqq 0$. Then $h_{p}^{1, l}=1$, if $l \leqq m$ and $h_{p}^{j, l}=0$, otherwise.

3) Let $p=-2 m+1, m \leqq 0$, Then $h_{p}^{0, l}=1$, if $l \geqq m$, and $h_{p}^{j, l}=0$, otherwise.

4) Let $p=-2 m+2, m \leqq 0$. Then $h_{p}^{-1, l}=1$, if $l \geqq m$ and $h_{p}^{j, l}=0$, otherwise.

Proof. Let $p=-2 m, m \geqq 0$. By Theorem $4, h_{p}^{j, l}=\sum_{k \geqq 0} h^{j}\left(L_{|l|+2 k} \otimes \mathscr{H}_{p+l}\right)$. It is equal to $\delta_{j, 0}$, if $l \leqq m$, and 0 , if $l>m$, by Proposition 7 .

In other cases the proof is similar. 
Since $d_{p}^{j, l}=h_{p}^{j, l}+h_{p}^{j-1, l}$ (cf. p. 628), Theorem 2 on p. 628 should read as follows.

Thcorem 2. 1) Let $p=-2 m, m \geqq 0$. Then $d_{p}^{0, l}=d_{p}^{1, l}=1$, if $l \leqq m$, and $d_{p}^{j, l}=0$, otherwise.

2) Let $p=-2 m-1, m \geqq 0$. Then $d_{p}^{1, l}=d_{p}^{2, l}=1$, if $l \leqq m$, and $d_{p}^{j, l}=0$, otherwise.

3) Let $p=-2 m+1, m \leqq 0$. Then $d_{p}^{0, l}=d_{p}^{1, l}=1$, if $l \geqq m$, and $d_{p}^{j, l}=0$, otherwise.

4) Let $p=-2 m+2, m \leqq 0$. Then $d_{p}^{0, l}=d_{p}^{-1, l}=1$, if $l \geqq m$, and $d_{p}^{j, l}=0$, otherwise.

\section{References}

1. Feigin, B., Fuchs, D.: Representations of the Virasoro algebra. In: Representations of Lie groups and related topics. Vershik, A.M., Zhelobenko, D.P. (eds.), pp. 465-554. New York: Gordon and Breach 1990

2. Frenkel, E.: Determinant formulas for the free field representations of the Virasoro and KacMoody algebras. Harvard Preprint, February 1992. Submitted to Phys. Lett. B

Communicated by A. Jaffe 